\title{
Flow invariant subsets for geodesic flows of manifolds with non-positive
} curvature

\author{
Reinold, B
}

\begin{abstract}
Consider a closed, smooth manifold $\mathrm{M}$ of non-positive curvature. Write $\mathrm{p}: \mathrm{UM} \rightarrow \mathrm{M}$ for the unit tangent bundle over $\mathrm{M}$ and let $>$ denote the subset consisting of all vectors of higher rank. This subset is closed and invariant under the geodesic flow on UM. We define the structured dimension s-dim > which, essentially, is the dimension of the set $\mathrm{p}(>)$ of base points of $>$. The main result of this paper

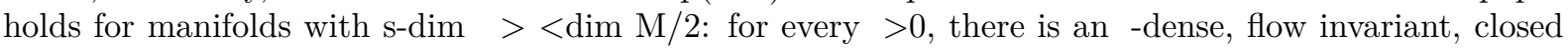
subset $\Xi \quad \mathrm{UM}>$ such that $\mathrm{p}(\Xi)=\mathrm{M}$.
\end{abstract}

DOI: https://doi.org/10.1017/S0143385704000197

Posted at the Zurich Open Repository and Archive, University of Zurich ZORA URL: https://doi.org/10.5167/uzh-21817

Journal Article

Originally published at:

Reinold, B (2004). Flow invariant subsets for geodesic flows of manifolds with non-positive curvature. Ergodic Theory and Dynamical Systems, 24(6):1981-1990.

DOI: https://doi.org/10.1017/S0143385704000197 


\title{
Flow invariant subsets for geodesic flows of manifolds with non-positive curvature
}

\author{
BERT REINOLD \\ Institut für Mathematik, Universität Zürich, Winterthurer Str. 190, CH-8057 Zürich, \\ Switzerland \\ (e-mail: reinold@math.unizh.ch)
}

(Received 16 November 2003 and accepted in revised form 4 February 2004)

\begin{abstract}
Consider a closed, smooth manifold $M$ of non-positive curvature. Write $p: U M \rightarrow M$ for the unit tangent bundle over $M$ and let $\mathcal{R}_{>}$denote the subset consisting of all vectors of higher rank. This subset is closed and invariant under the geodesic flow $\phi$ on $U M$. We will define the structured dimension s-dim $\mathcal{R}_{>}$which, essentially, is the dimension of the set $p\left(\mathcal{R}_{>}\right)$of base points of $\mathcal{R}_{>}$.

The main result of this paper holds for manifolds with s-dim $\mathcal{R}_{>}<\operatorname{dim} M / 2$ : for every $\epsilon>0$, there is an $\epsilon$-dense, flow invariant, closed subset $\Xi_{\epsilon} \subset U M \backslash \mathcal{R}_{>}$such that $p\left(\Xi_{\epsilon}\right)=M$.
\end{abstract}

\section{Introduction}

The aim of this paper is to generalize a result $\dagger$ of Burns and Pollicott for manifolds of constant negative curvature to the case of non-positively curved manifolds of rank one. They showed that, on a compact surface $M$ of constant negative curvature, it is possible to construct a closed proper subset $\Xi$ of the unit tangent bundle $U M$ which is invariant under the geodesic flow and full in the sense that its image under the base-point projection $p(\Xi)$ is the whole surface $M$. This means that there is an open subset $\mathcal{W}$ of $U M$ such that, for every point $p$ in $M$, we can find a geodesic passing through $p$ such that the velocity field of the geodesic avoids that subset $\mathcal{W}$. By the construction, $\mathcal{W}$ was always some neighbourhood of a non-recurrent vector.

For dimension bigger than three, Schroeder improved this result to manifolds with curvature $K<-1$ in [7]. He even proved that a neighbourhood like $\mathcal{W}$ can be found for every vector, no matter whether it is recurrent or not.

The next generalization took place in [3]. Buyalo and Schroeder considered a compact manifold of rank one and proved the existence of a neighbourhood like $\mathcal{W}$ for every vector of rank one.

The result of this paper is that even for vectors of higher rank a neighbourhood $\mathcal{W}$ can be avoided, provided that the set of vectors of higher rank satisfies some dimensional condition. The main result is the following.

$\dagger$ The proof is unpublished but the result is stated in [4]. 
THEOREM 1.1. Let $M$ be a compact manifold of non-positive curvature. Suppose the s-dimension of the set of vectors of higher rank $\mathcal{R}_{>}$is bounded by

$$
\text { s-dim } \mathcal{R}_{>}<\frac{\operatorname{dim} M}{2}
$$

Then, for every $\epsilon>0$, there is a closed, flow invariant, full, $\epsilon$-dense subset $\Xi_{\epsilon}$ of the unit tangent bundle UM consisting only of vectors of rank one.

This result was obtained as part of the author's thesis [6]. The proof as presented in this paper has been considerably shortened to explain the main ideas. For further details, please refer to the original thesis.

\section{Notation}

Throughout this text, $M$ will denote a complete, compact, Riemannian manifold of nonpositive curvature. The unit tangent bundle with the base-point projection is denoted by $p: U M \rightarrow M$. The Riemannian metric on $M$ induces a Riemannian metric (the Sasaki metric) on the unit tangent bundle $U M$. Both metrics induce distances, denoted by $d(\cdot, \cdot)$. With respect to the metric on $U M$, we will write $\mathcal{W}_{\epsilon}(v)$ for an $\epsilon$-neighbourhood of a vector $v \in U M$.

For an element $v \in U M, \gamma_{v}$ denotes the unique geodesic with initial condition $\dot{\gamma}_{v}(0)=v$. The geodesic flow $\phi_{t}$ is defined by $\phi_{t}(v):=\dot{\gamma}_{v}(t)$. We will say that a geodesic $\gamma$ avoids an $\eta$-neighbourhood of the vector $v \in U M$, if $d(\dot{\gamma}, v) \geq \eta$, i.e. the distance of $v$ to the velocity field $\dot{\gamma}$ is bounded below by $\eta$.

For a geodesic $\gamma$, the set of all parallel Jacobi fields along that geodesic is a vector space. By $\operatorname{rank}(\gamma)$, we denote the dimension of this vector space. For a unit vector $v$, define $\operatorname{rank}(v):=\operatorname{rank}\left(\gamma_{v}\right)$. The rank of a manifold is defined to be the minimal rank of its tangent vectors. From a result by Ballmann [2, Appendix 1], closed manifolds of rank greater than one are quotients of either products or locally symmetric spaces. We will, therefore, always suppose that $M$ is of rank one, i.e. there is a geodesic $\gamma$ such that the only parallel Jacobi fields along $\gamma$ are multiples of its velocity field $\dot{\gamma}$. The set of all vectors of rank one is denoted by $\mathcal{R}_{1}$, and the set of all vectors of higher rank by $\mathcal{R}_{>}$. Both these sets are flow invariant; $\mathcal{R}_{1}$ is open, $\mathcal{R}_{>}$closed. If $M$ is a real analytic manifold, then both sets are subanalytic (see [5] for a definition). This motivates the definition of the structured dimension.

For any subset $\mathcal{R} \subset U M$, a support of $\mathcal{R}$ is a finite union $Z=\bigcup Z_{i}$ of closed submanifolds $Z_{i} \subset M$ (called the strata of $Z$ ) such that $\mathcal{R} \subset U Z:=\bigcup U Z_{i}$, i.e. all elements of $\mathcal{R}$ are tangent to some stratum in $Z$. The dimension of a support is defined to be the maximal dimension of one of its strata. By s-dim $\mathcal{R}$ we denote the minimal dimension of a support of $\mathcal{R}$ and call this the structured dimension of $\mathcal{R}$. A support $Z$ of $\mathcal{R}$ is called an $s$-support of $\mathcal{R}$ if $\operatorname{dim} Z=s$-dim $\mathcal{R}$, i.e. if $Z$ realizes the structured dimension of $\mathcal{R}$. Note that $0 \leq \mathrm{s}-\operatorname{dim} \mathcal{R} \leq \operatorname{dim} M$ for any subset of $U M$. For example, for a submanifold $Y \subset M$, the unit tangent bundle $U Y$ has structured dimension s-dim $U Y=\operatorname{dim} Y$. From now on, $Z$ will always denote an s-support of $\mathcal{R}_{>}$.

$\pi: X \rightarrow M$ is the universal covering of $M$ by the Hadamard manifold $X$. The covering maps $\pi: X \rightarrow M$ and $d \pi: U X \rightarrow U M$ induce Riemannian metrics on $X$ and $U X$. 
With respect to these metrics, geodesics are mapped to geodesics and the rank does not change. Therefore, we will use the same notation in $X$ as in $M$. Throughout this paper, we will mostly work with $X$ but the fact that $X$ has the compact quotient $M$ will be essential.

We will be concerned with some structures in the unit tangent spaces which we will call spheres and denote by $\mathcal{S}_{r}(p)$. If $S_{r}(p)$ is the sphere of radius $r$ around the point $p$ in $X$, then the set of outward normal vectors to this sphere will be denoted by $\mathcal{S}_{r}(p)$. Another way to describe this set is via the identity $\mathcal{S}_{r}(p)=\phi_{r}\left(U_{p} X\right)$. For fixed $r \geq 0$, the unit tangent bundle is foliated into these spheres. For $r \rightarrow \infty$, the spheres $\mathcal{S}_{r}\left(\gamma_{v}(-r)\right)$ converge to the strong unstable leaf of $v$, i.e. to the outward pointing normal field to the horosphere at $\gamma_{v}(-\infty)$ through $p(v)$. If we only consider $\epsilon$-neighbourhoods of $v$ inside these spheres, then the convergence is uniform.

\section{Construction}

We want to construct a closed, flow invariant, $\epsilon$-dense, subset $\Xi_{\epsilon}$ of $U M$ which consists only of vectors of rank one and is full in the sense that $p\left(\Xi_{\epsilon}\right)=M$. For fixed $\eta$, define a flow invariant, closed set of rank one vectors by

$$
\hat{\Xi}_{\eta}:=\overline{\left\{u \in U M \mid \gamma_{u} \text { avoids an } \eta \text {-neighbourhood of } \mathcal{R}_{>}\right\}} .
$$

We will see that, for $\eta=\eta(\epsilon)$ small enough, the set $\Xi_{\epsilon}:=\hat{\Xi}_{\eta}$ is $\epsilon$-dense and full in the previous sense. For the moment, we will not focus on the fact that $\Xi_{\epsilon}$ is $\epsilon$-dense. To prove that $\Xi_{\epsilon}$ is full, we construct, for every point $o \in M$, a geodesic $\gamma$ through $o$ which avoids the $\eta$-neighbourhood of $\mathcal{R}_{>}$. Then $\dot{\gamma}(0) \in \Xi_{\epsilon}$ and, hence, $o \in p\left(\Xi_{\epsilon}\right)$.

For the construction, we work in $U X$. Given a starting point $o \in X$, begin by constructing a sequence $v_{i}$ of vectors in $U_{o} X$ such that the geodesic segments $\left.\gamma_{v_{i}}\right|_{\left[0, t_{i}\right]}$ avoid a neighbourhood of $\mathcal{R}_{>}$and $t_{i} \rightarrow \infty$. By the hyperbolicity $\dagger$ of the vectors of rank one, the limit $v_{\infty}:=\lim v_{i}$ will exist and the geodesic ray $\gamma_{v_{\infty}} \mid \mathbb{R}_{+}$will avoid a neighbourhood of $\mathcal{R}_{>}$.

Now instead of just considering one vector $v_{0}$, consider a compact, low-dimensional manifold $Y$ together with a smooth map $V_{0}: Y \rightarrow U_{o} X$ and define $t_{-1}:=0$. Recursively, we define a sequence of smooth maps $V_{i}: Y \rightarrow U_{o} X$ and times $t_{i} \rightarrow \infty$, such that the $V_{i}$ converge to a continuous map $V_{\infty}$ sufficiently close to our original map $V_{0}$.

Suppose $V_{i}$ and $t_{i-1}$ are given. We define

$$
t_{i}:=\min \left\{t \geq t_{i-1}+B \mid d\left(\phi_{t} \circ V_{i}(Y), U Z\right) \leq 11 \epsilon\right\}
$$

and deform $V_{i}$ into $V_{i+1}$ as follows, where $C$ and $\tau$ are constants provided by Lemma 4.1. Deform the smooth map $\phi_{t_{i}} \circ V_{i}: Y \rightarrow \mathcal{S}_{t_{i}}(o)$ slightly, as explained in Proposition 4.1, to a $C$-close map into $\mathcal{S}_{t_{i}}(o)$ whose image is $\tau$-far away from $U Z$. Composing the resulting map with $\phi_{-t_{i}}$ gives a map $V_{i+1}: Y \rightarrow U_{o} X$. If $t_{i}=\infty$ for some $i$, then define $V_{j}:=V_{i}$ and $t_{j}:=t_{i-1}+(j-i+1) B$ for all $j \geq i$. By this construction, we have the following immediate properties.

(1) The image of $\phi_{t_{j}} \circ V_{j+1}$ is $\tau$-far from $U Z$ (and, hence, from $\mathcal{R}_{>}$). We say that $V_{j+1}$ is $\tau$-far from $U Z$ at time $t_{j}$.

$\dagger$ Compare this with $\S 5$. 
(2) The maps $\phi_{t_{j}} \circ V_{j}$ and $\phi_{t_{j}} \circ V_{j+1}$ are $C$-close. We say that $V_{j}$ and $V_{j+1}$ are $C$-close at time $t_{j}$.

(3) Two times $t_{j}$ and $t_{j+1}$ of deformations are separated by at least time $B$, i.e. the deformations happen at discrete, distant times.

In $\S 5$, we will see that, by (3), the widening property of the vectors of rank one will guarantee that the deformation at time $t_{j}$ will not displace the image of $\phi_{t} \circ V_{j}$ much for earlier times $0<t<t_{j}$. Therefore, the geodesic rays $\gamma_{V_{j}}$ will avoid $\mathcal{R}_{>}$for longer and longer times and the smooth maps $V_{j}: Y \rightarrow U_{o} X$ will converge to a continuous map $V_{\infty}: Y \rightarrow U_{o} X$ for $j \rightarrow \infty$.

\section{Perturbations on the Spheres $\mathcal{S}_{r}$}

Given the s-support $Z$, we want to describe a deformation $\Psi_{r}$ of $U X$ that respects the $r$-sphere foliation (i.e. $\Psi_{r}\left(\mathcal{S}_{r}(o)\right) \subset \mathcal{S}_{r}(o)$ for all $r>r_{0}$ and $\left.o \in X\right)$ and such that the displacement by $\Psi_{r}$ is small but moves every vector away from $U Z$ effectively. For the moment, suppose that $Z$ consists of only one stratum, hence $Z$ is a closed submanifold of $U M$. By the remark at the end of this section, we will see that this restriction has no effect on Proposition 4.1.

For every time $r>r_{0}$ and small $\epsilon$, we define a perturbation $\Psi_{r, \epsilon}$ of $U X \backslash p^{-1} Z$ that respects the foliation of $U X$ into spheres of radius $r$ and such that the distance of the image of $\Psi_{r, \epsilon}$ to $U Z$ is at least $\epsilon$. The idea is illustrated in Figure 1 and works as follows. For any vector $v \in U X \backslash p^{-1} Z$ with $d(v, U Z)<4 \epsilon$, let $\gamma$ denote the shortest geodesic $\dagger$ from $Z$ to $p:=p(v)$. Use parallel transport along $\gamma$ to move $v$ away from $Z$ to a point $q$ which is at distance $2 \epsilon-d(v, U Z) / 2$ and write $\bar{v}$ for this vector. Find the vector $w$ in $U_{q} X$ such that $\gamma_{w}\left(\mathbb{R}_{-}\right)$goes through $o:=\gamma_{v}(-r)$ (hence, $o$ is the centre of the sphere containing $v$ ). Pull back $w$ by the geodesic flow to a vector on $\mathcal{S}_{r}(o)$ and call this image $\Psi_{r, \epsilon}(v)$.

For $v$ far away from $U Z$, the vector is only slightly displaced and, hence, stays away from $U Z$. For $v$ close to $U Z$, the parallel transport moves the vector very effectively away from $U Z$. The readjustment afterwards is comparatively small and, hence, the resulting vector is far away from $U Z$. This is summed up in the following lemma.

LEMMA 4.1. Given $r_{0}>0$, we can find constants $C, \tau, \epsilon$ such that for all $\left.\left.\lambda \in\right] 0,1\right]$ the maps $\Psi_{r, \lambda \epsilon}$ have the following properties for all $r \geq r_{0}$ :

(1) $\Psi_{r, \lambda \epsilon}: U X \backslash p^{-1} Z \rightarrow U X$ is continuous and respects the $r$-sphere foliation of $U X$.

(2) The image of $\Psi_{r, \lambda \epsilon}$ has no intersection with the $\lambda \tau$-neighbourhood of UZ:

$$
d\left(\Psi_{r, \lambda \epsilon}(v), U Z\right) \geq \lambda \tau \quad \text { for all } v \in U X \backslash p^{-1} Z .
$$

(3) The displacement by $\Psi_{r, \lambda \epsilon}$ is bounded by the global constant $\lambda C$ :

$$
d\left(\Psi_{r, \lambda \epsilon}(v), v\right) \leq \lambda C \quad \text { for all } v \in U X \backslash p^{-1} Z .
$$

Remark. If $Z$ is a manifold with boundary, some problems might arise at the boundary, since the parallel transport could point in the same direction as the geodesic flow and, hence, will be undone in the last step of the construction. However, these problems can be overcome if we use a slightly bigger submanifold $Z^{\prime} \supset Z$ for the construction of $\Psi_{r, \epsilon}$. Hence, Lemma 4.1 holds in this case, too.

$\dagger$ If $\epsilon$ is small enough, $\gamma$ is unique. 




FIGURE 1. Construction of $\Psi_{r, \epsilon}$.

As a result we can deform submanifolds of spheres away from a stratum $Z$.

Proposition 4.1. For $r_{0}>0$ given, there are constants $C, \tau>0$ such that for any $\lambda \in] 0,1], r>r_{0}, o \in X$ and manifold $Y$ with

$$
\operatorname{dim} Y<\operatorname{dim} X-\operatorname{dim} Z
$$

we can deform any smooth map $c: Y \rightarrow \mathcal{S}_{r}(o)$ into a smooth map $c_{\lambda}: Y \rightarrow \mathcal{S}_{r}(o)$ such that

- $\quad c_{\lambda}$ is $\lambda$ C-close to $c$, i.e.

$$
d\left(c(x), c_{\lambda}(x)\right) \leq \lambda C \quad \text { for all } x \in Y .
$$

- $\quad c_{\lambda}$ avoids a $\lambda \tau$-neighbourhood of $U Z$, i.e.

$$
d\left(c_{\lambda}(x), U Z\right) \geq \lambda \tau \quad \text { for all } x \in Y .
$$

Proof. Choose $\tilde{C}, \tilde{\epsilon}$ and $\tilde{\tau}$ as provided by Lemma 4.1 for $r_{0} / 2$. Without loss of generality, we can assume that $\tilde{\tau}<r_{0} / 2$ since the constants are scalable. Fix these constants and consider any $r \geq r_{0}$ and $\left.\left.\lambda \in\right] 0,1\right]$. Choose $\tau^{\prime}>0$ such that on big spheres (radius $>r_{0} / 2$ ) vectors are $\tilde{\tau} \lambda / 6$-close if their base points are $\tau^{\prime}$-close. Now fix $o \in X$ and consider the differentiable map

$$
\begin{aligned}
\rho: Z \backslash\{o\} & \longrightarrow \mathbb{R} \\
p & \longmapsto d(o, p),
\end{aligned}
$$


which measures the radius in polar coordinates around $o$. By Sard's theorem, the set of regular values of $\rho$ is dense in $\mathbb{R}$. Hence, we can choose a regular value $\left.r^{\prime} \in\right] r-\tilde{\tau} \lambda / 6, r+\tilde{\tau} \lambda / 6\left[\right.$. The inverse image $Z_{r^{\prime}}:=\rho^{-1}\left(r^{\prime}\right)$ is the intersection of $Z$ with the sphere $S_{r^{\prime}}(o)$ in $X$. Since $r^{\prime}$ is regular, this intersection $Z_{r^{\prime}}$ is a smooth submanifold of $\operatorname{dimension}$ at most $\operatorname{dim} Z-\operatorname{dim} \mathbb{R}=\operatorname{dim} Z-1$ in the sphere $S_{r^{\prime}}(o)$ by the Preimage Theorem. Next note that the geodesic flow $\phi_{r^{\prime}-r}$ identifies the two spheres $\mathcal{S}_{r}(o) \rightarrow \mathcal{S}_{r^{\prime}}(o)$, moving every vector by exactly $\left|r^{\prime}-r\right|<\tilde{\tau} / 6 \lambda$. Furthermore, we have natural identifications of spheres with centre $o$ in $X$ and in $U X$, given by the gradient of $\rho$ : $\left.\operatorname{grad} \rho\right|_{S_{r}(o)}: S_{r}(o) \rightarrow \mathcal{S}_{r}(o)$ and $\left.\operatorname{grad} \rho\right|_{S_{r^{\prime}}(o)}: S_{r^{\prime}}(o) \rightarrow \mathcal{S}_{r^{\prime}}(o)$.

Now $y:=p \circ \phi_{r^{\prime}-r} \circ c: Y \rightarrow S_{r^{\prime}}(o)$ is a smooth map of $Y$ into the sphere $S_{r^{\prime}}(o)$. By Thom's Transversality Theorem, we can find a $\tau^{\prime}$-close smooth map $y^{\prime}: Y \rightarrow S_{r^{\prime}}(o)$ which avoids $Z_{r^{\prime}}$. Write $\alpha$ for the homotopy of $S_{r^{\prime}}(o)$ with $\alpha_{0}=\operatorname{id}_{S_{r^{\prime}}(o)}$ and $\alpha_{1} \circ y=y^{\prime}$. Consider the map

$$
c_{\lambda}:=\phi_{r-r^{\prime}} \circ \Psi_{r, \lambda \epsilon} \circ \operatorname{grad} \rho \circ \alpha_{1} \circ p \circ \phi_{r^{\prime}-r} \circ c: Y \rightarrow \mathcal{S}_{r}(o) .
$$

This looks quite monstrous at first glance but we will explain it step by step.

- $\quad \phi_{r^{\prime}-r} \circ c$ is $\tilde{\tau} \lambda / 6$-close to $c$, since $r^{\prime}-r<\tilde{\tau} \lambda / 6$.

- The base-point distance between $\phi_{r^{\prime}-r} \circ c$ and $\operatorname{grad} \rho \circ \alpha_{1} \circ p \circ \phi_{r^{\prime}-r} \circ c$ is just the distance between $y$ and $y^{\prime}$, which is smaller than $\tau^{\prime}$ by definition of $\alpha$. But $\tau^{\prime}$-close base points imply $\tilde{\tau} \lambda / 6$-close radial vectors and, hence, $\operatorname{grad} \rho \circ \alpha_{1} \circ p \circ \phi_{r^{\prime}-r} \circ c=$ $\operatorname{grad} \rho \circ y^{\prime}$ is $\tilde{\tau} \lambda / 6$-close to $\phi_{r^{\prime}-r} \circ c$ and $\tilde{\tau} \lambda / 3$-close to $c$.

- $\quad y^{\prime}$ avoids $Z$ and, hence, $\operatorname{grad} \rho \circ y^{\prime}$ maps $Y$ into $U X \backslash p^{-1} Z$ and, hence, the map $\Psi_{r, \lambda \epsilon} \circ \operatorname{grad} \rho \circ y^{\prime}$ is well defined. By Lemma 4.1, it is $\tilde{C} \lambda$-close to $\operatorname{grad} \rho \circ y^{\prime}$ and, hence, $(\tilde{C} \lambda+\tilde{\tau} \lambda / 3)$-close to our original map $c$. Again by Proposition 4.1, it avoids a $\tilde{\tau} \lambda$-neighbourhood of $U Z$.

- $\quad$ Projecting this map back to $\mathcal{S}_{r}(o)$ by the geodesic flow $\phi_{r-r^{\prime}}$ gives a further displacement of $\left|r-r^{\prime}\right| \leq \tilde{\tau} \lambda / 6$ which leaves us with the properties that $c_{\lambda}$ is $(\tilde{C} \lambda+\tilde{\tau} \lambda / 3+\tilde{\tau} \lambda / 6)$-close to $c$ and avoids a $(\tilde{\tau} \lambda-\tilde{\tau} \lambda / 6)$-neighbourhood of $U Z$.

This ends the proof if we set $C:=\tilde{C}+\tilde{\tau} / 2$ and $\tau:=\tilde{\tau} / 2$.

Remark. Proposition 4.1 stays true if $Z$ is not a submanifold of $M$ but a finite union $Z=$ $\bigcup Z_{i}$ of closed submanifolds of $M$ and $\operatorname{dim} Y<\operatorname{dim} X-\max \operatorname{dim} Z_{i}$. To see this, pick $C_{i}, \tau_{i}$ for each of the submanifolds and then choose $\lambda_{i+1}$ so small that $\lambda_{i+1} C_{i+1}<\tau_{i} / 8$ and $\lambda_{i+1} \tau_{i+1}<\tau_{i} / 4$. Now, after a finite number of displacements, we avoid all $Z_{i}$ since the $(i+1)$ st displacement will not undo the previous ones.

\section{Hyperbolicity}

It is a well-known fact that, in hyperbolic space, geodesics originating in the same point diverge qualitatively faster than in Euclidean space. Ballmann [1] introduced the term hyperbolic geodesic for geodesic segments where the distance between close geodesic segments is less than $\mu$ times the Hausdorff distance for some $\mu \in] 0,1[$. Buyalo and Schroeder [3] use a similar definition to define a hyperbolic vector and show that every vector of rank one is, indeed, hyperbolic. As a result, a compact set of rank-one vectors has a widening property explained in the following lemma and illustrated in Figure 2. 


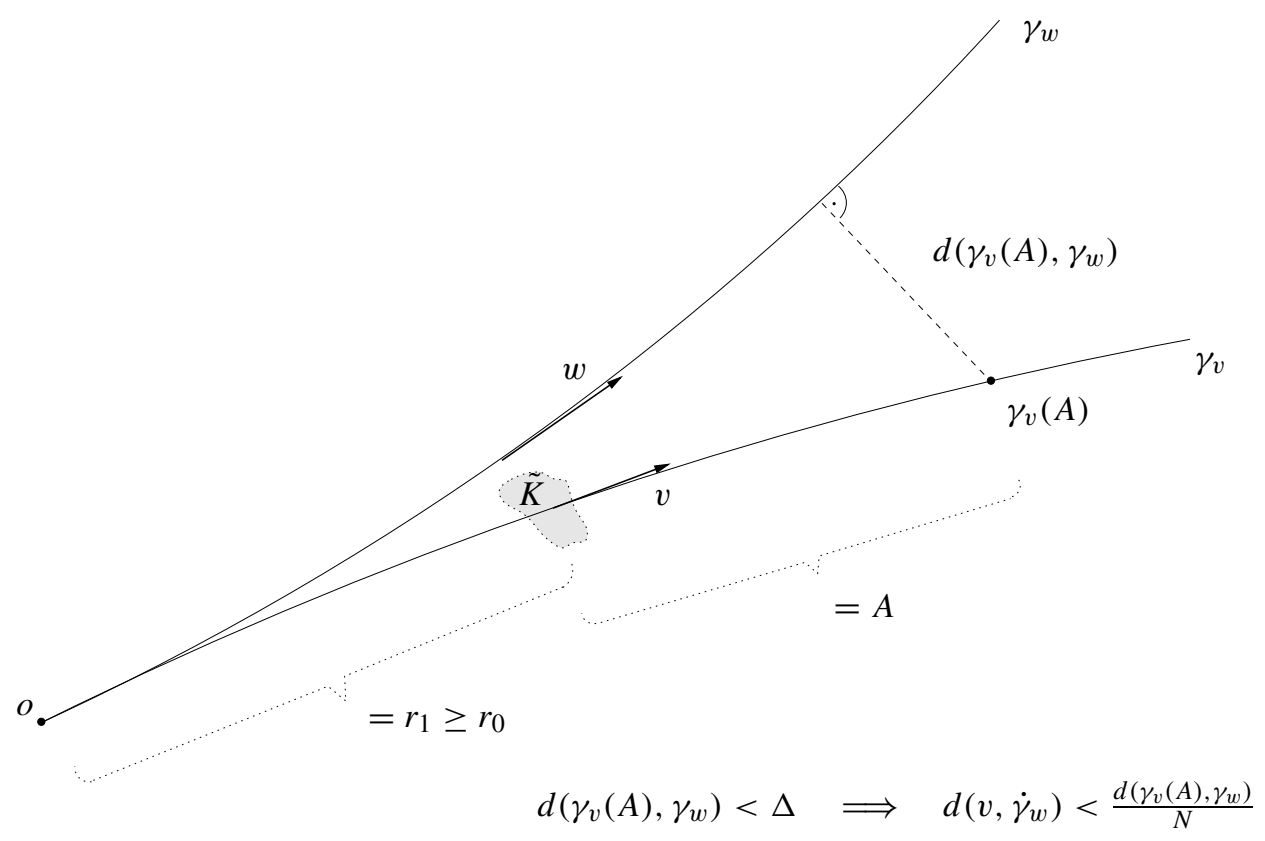

FIGURE 2. Widening property of $\tilde{K}$.

LEMMA 5.1. Consider a compact set $K \subset U M$ consisting of vectors of rank one and $\tilde{K}:=d \pi^{-1} K$. Given $N \in \mathbb{N}$ and $\Delta, r_{0}>0$, there is a distance $A>0$ such that for any point $o \in X$ and vectors $v \in \tilde{K}$ and $w \in U X$ with $\gamma_{v}\left(-r_{1}\right)=o=\gamma_{w}\left(-r_{2}\right)\left(r_{1}>r_{0}\right.$, $\left.r_{2}>0\right)$, the inequality $d\left(\gamma_{v}(A), \gamma_{w}\right) \leq \Delta$ implies $d\left(v, \dot{\gamma}_{w}\right)<d\left(\gamma_{v}(A), \gamma_{w}\right) / N$.

The important point here is that $A=A\left(K, N, \Delta, r_{0}\right)$ does not depend on the time $r_{1}$. To illustrate the widening property, suppose $K, N, \Delta, r_{0}$ are given and $A$ is the constant provided by the lemma. Consider two close geodesics $\gamma$ and $\sigma$ starting in $o \in X$. Suppose that outside the ball of radius $r_{0}$ around $o$ there is a point $\gamma(t)\left(t>r_{0}\right)$, where the tangent vector $v:=\dot{\gamma}(t)$ to $\gamma$ lies in $\tilde{K}$. Now if $\sigma$ meets the ball of radius $\Delta$ around the point $\gamma(t+A)$, then the velocity field $\dot{\sigma}$ is $\Delta / N$-close to $v$.

Roughly speaking, we can say that $A$ is the time span after which the distance between close geodesics widens by a factor of $N$. Note that, in Euclidean space, it is impossible to find such $A$ which works for all times $t>r_{0}$, since the distance between geodesics grows linearly.

\section{Choosing the right constants}

Suppose that s-dim $\mathcal{R}_{>}<\operatorname{dim} M-1$ and $Z$ is an s-support of $\mathcal{R}_{>}$. Fix $r_{0}>2$ and find constants $\tau, C$ by Proposition 4.1 for $Z$. Define new constants

$$
\epsilon:=\frac{\tau}{10} \quad \text { and } \quad \delta:=10 \frac{C}{\tau},
$$

fix $N \in \mathbb{N}$ such that $N>1+\frac{1}{2} \delta$, fix

$$
\frac{\delta}{N-1}<\Lambda<2 N-\delta
$$






FIGURE 3. Construction of $v_{i+1, i}$ and $t_{i+1, k}$.

and choose $\Delta>(\delta+\Lambda) \epsilon$. Since Proposition 4.1 holds for $\lambda \tau$ and $\lambda C$ if $\lambda \in] 0,1]$, we can use the same constants $\delta, N, \Lambda$ and $\Delta$ when working with $\epsilon^{\prime}<\epsilon$. Fix $A$ as provided by Lemma 5.1 for the compact set $K_{\epsilon}:=U M \backslash \mathcal{W}_{\epsilon}(U Z)$ and $B>A+8 \epsilon$. Note that, for all $t \in \mathbb{R}$, the compact set $K_{\epsilon}$ consists only of vectors of rank one while the compact set $\mathcal{R}_{>}$ consists of vectors of higher rank. Hence, these sets are disjoint and we can define

$$
\beta:=\min _{t \in[-B, B]} d\left(\phi_{t}\left(K_{\epsilon}\right), \mathcal{R}_{>}\right) / \epsilon
$$

Given a smooth map $V_{0}: Y \rightarrow U_{o} X$, we construct the sequences $t_{i} \in \mathbb{R}_{+}$and $V_{i}: Y \rightarrow U_{o} X$ as explained in $\S 3$. We consider one point $x \in Y$ and its images $v_{i}=V_{i}(x) \in U_{o} X$.

As illustrated in Figure 3, define $t_{i, k}$ and $v_{i, k}$ for $0 \leq k \leq i-1$ by the equation

$$
d\left(\phi_{t_{i, k}}\left(v_{i}\right), \phi_{t_{k}}\left(v_{k+1}\right)\right)=d\left(\dot{\gamma}_{v_{i}}, \phi_{t_{k}}\left(v_{k+1}\right)\right)
$$

i.e. $v_{i, k}:=\phi_{t_{i, k}}\left(v_{i}\right)$ is the vector, tangent to the geodesic $\gamma_{v_{i}}$ which is closest to the vector $v_{k, k}:=\phi_{t_{k}}\left(v_{k+1}\right)$. Now we know that

$$
\begin{gathered}
\beta \epsilon<d\left(v_{i, i}, \mathcal{R}_{>}\right) \\
\quad d\left(v_{i+1, i}, v_{i, i}\right) \leq \delta \epsilon \\
10 \epsilon \leq d\left(v_{i+1, i}, \mathcal{R}_{>}\right)
\end{gathered}
$$


and, step by step, we can prove that $v_{i+1, i}=v_{i} \in K_{\epsilon}$,

$$
\begin{array}{cl}
\beta \epsilon \leq d\left(\phi_{t}\left(v_{i}\right), \mathcal{R}_{>}\right) & \text {for } t_{i-1}-B \leq t \leq t_{i-1}+B \\
11 \epsilon<d\left(\phi_{t}\left(v_{i}\right), U Z\right) & \text { for } t_{i-1}+B \leq t<t_{i}
\end{array}
$$

and, for $0 \leq k<i$,

$$
\begin{aligned}
d\left(v_{i, k}, v_{k, k}\right) & <(\delta+\Lambda) \epsilon \\
d\left(v_{i, k}, v_{k+1, k}\right) & <2 \epsilon
\end{aligned}
$$

and $v_{i, k} \in K_{\epsilon}$. Furthermore,

$$
t_{i, i}:=t_{i}=t_{i, i-1} \quad \text { and } \quad\left|t_{k}-t_{i, k}\right| \leq 4 \epsilon
$$

and, finally,

$$
\begin{gathered}
\beta \epsilon<d\left(\phi_{t}\left(v_{i}\right), \mathcal{R}_{>}\right) \quad \text { for } 0 \leq t \leq t_{i}(i \geq 1) \\
d\left(v_{i+1, i-k}, \dot{\gamma}_{v_{i}}\right)<\frac{\delta}{N^{k}} \epsilon .
\end{gathered}
$$

By (2), the $v_{i}$ converge to some $v_{\infty}$ with

$$
d\left(v_{0}, v_{\infty}\right) \leq \frac{\delta}{N(N-1)} \epsilon
$$

and by (1) for $v_{\infty}$, we have

$$
\beta \epsilon \leq d\left(\dot{\gamma}_{v_{\infty}}\left(\mathbb{R}_{+}\right), \mathcal{R}_{>}\right) .
$$

Since none of the estimations depended on $v_{0}$, the convergence is uniform on $Y$ and, hence, the maps $V_{i}: Y \rightarrow U_{o} X$ converge to a continuous map $V_{\infty}: Y \rightarrow U_{o} X$. Now we can prove the following proposition.

Proposition 6.1. There are constants $\epsilon$ and $c$ such that, for any $\eta<\epsilon$, there is an $\eta^{\prime}<\eta$ such that, for any compact manifold $Y$ with

$$
\operatorname{dim} Y<\operatorname{dim} X-\mathrm{s}-\operatorname{dim} \mathcal{R}_{>}
$$

for any $o \in X$ and any continuous map $V_{0}: Y \rightarrow U_{o} X$, we can find a map $V_{\infty}: Y \rightarrow U_{o} X$ which is c $\eta$-close to $V_{0}$ and defines a family of geodesic rays avoiding an $\eta^{\prime}$-neighbourhood of $\mathcal{R}_{>}$, i.e.

$$
d\left(\dot{\gamma}_{V_{\infty}(Y)}\left(\mathbb{R}_{+}\right), \mathcal{R}_{>}\right) \geq \eta^{\prime} .
$$

Proof. Take all the constants we had before. Note that, by definition, $\delta$ and $N$ are independent of $\epsilon$. So we can define the global constant $c:=\delta / N(N-1)$. Choose $\beta$, $B, A$ and $\Delta$ replacing $\epsilon$ by $\eta<\epsilon$. Write $\eta^{\prime}:=\beta \eta$ to get the desired result.

For the special case where $\operatorname{dim} Y=0$ we get the following corollary.

COROllary 6.1. Let $M$ denote a compact manifold of non-positive curvature with $\mathrm{s}-\operatorname{dim} \mathcal{R}_{>}<\operatorname{dim} M$. Then there is an $\eta^{\prime}>0$ such that, for every point $o \in M$, we can find a geodesic ray $\gamma$, starting in o whose tangent field avoids an $\eta^{\prime}$-neighbourhood of the set $\mathcal{R}_{>}$of all vectors of higher rank. 


\section{From rays to geodesics}

In Proposition 6.1, choose $\eta<\epsilon / c$. To find a complete geodesic, consider two complementary submanifolds $N_{1}$ and $N_{2}$ of $U_{o} X$ which intersect in exactly two antipodal points. Deform the inclusion $N_{1} \hookrightarrow U_{o} X$ of one of them so that it avoids $\mathcal{R}_{>}$for all $r>0$ and the inclusion $N_{2} \hookrightarrow U_{o} X$ of the other one so that it avoids $\mathcal{R}_{>}$for all $r<0$. If $\epsilon$ is smaller than $\pi$, then the images of the deformed maps still intersect in at least two points. Pick one of these points of intersection. It defines a complete geodesic $\gamma_{o}$ which avoids $\mathcal{R}_{>}$ at all times. The deformation of the $N_{i}$ works if $\operatorname{dim} N_{i}<\operatorname{dim} X-\mathrm{s}-\operatorname{dim} \mathcal{R}_{>}$. We need two complementary submanifolds, i.e. $\operatorname{dim} N_{1}+\operatorname{dim} N_{2}=\operatorname{dim} U_{o} X=\operatorname{dim} M-1$. Combining these two inequalities we see that the construction works if s-dim $\mathcal{R}_{>}<\operatorname{dim} M / 2$. Since the choice of constants is independent of $o$, we can find such $\gamma_{o}$ for all $o \in X$. The preimage under $d \pi$ of the union of the velocity fields of all such geodesics

$$
d \pi^{-1}\left(\bigcup_{o \in X} \dot{\gamma}_{o}\right)
$$

is full and contained in $\hat{\boldsymbol{\Xi}}_{\eta^{\prime}}$. Hence, $\hat{\Xi}_{\eta^{\prime}}$ is full. To see that $\hat{\boldsymbol{\Xi}}_{\eta^{\prime}}$ is $\epsilon$-dense, note that for any $v \in U_{o} X$ we can choose $N_{1}$ and $N_{2}$ such that they intersect in $v$ and $-v$. Then $\epsilon$-close to $v$ and $-v$ the images of the deformed maps will intersect and we can suppose that $d\left(\dot{\gamma}_{o}(0), v\right)<\epsilon$. Theorem 1.1 follows.

Acknowledgement. Most ideas in this paper originated in stimulating discussions with my supervisor Viktor Schroeder. I am deeply indebted to him for raising my interest in this subject and for many helpful remarks. The definition of the structured dimension is due to him and without this notion the result of this paper would have been hardly communicable.

\section{REFERENCES}

[1] W. Ballmann. Lectures on Spaces of Non-positive Curvature. Birkhäuser, Basel, 1995. With an appendix by Misha Brin.

[2] W. Ballmann, M. Gromov and V. Schroeder. Manifolds of Non-positive Curvature (Progress in Mathematics, 61). Birkhäuser, Boston, MA, 1985.

[3] S. Buyalo and V. Schroeder. Invariant subsets of rank 1 manifolds. Manuscripta Math. 107(1) (2002), 73-88.

[4] S. Buyalo, V. Schroeder and M. Walz. Geodesics avoiding open subsets in surfaces of negative curvature. Ergod. Th. \& Dynam. Sys. 20(4) (2000), 991-1006.

[5] H. Hironaka. Subanalytic sets. Number Theory, Algebraic Geometry and Commutative Algebra, in Honor of Yasuo Akizuki. Kinokuniya, Tokyo, 1973, pp. 453-493.

[6] B. Reinold. Vectors of higher rank on a Hadamard manifold with compact quotient. Dissertation, Universität Zürich, 2003. arXiv:math.DG/0311025.

[7] V. Schroeder. Bounded geodesics in manifolds of negative curvature. Math. Z. 235(4) (2000), 817-828. 